\title{
Coincidence and Fixed Point of Nonexpansive Type Mappings in 2-Metric Spaces
}

\author{
H. A. Hammad ${ }^{1, *}$, A. H. Ansari ${ }^{2}$ and R. A. Rashwan ${ }^{3}$ \\ ${ }^{1}$ Department of Mathematics, Faculty of Science, Sohag University, Sohag 82524, Egypt \\ e-mail: h_elmagd89@yahoo.com \\ ${ }^{2}$ Department of Mathematics, Karaj Branch, Islamic Azad University, Karaj, Iran \\ e-mail: dssaluja@ rediffmail.com \\ ${ }^{3}$ Department of Mathematics, Faculty of Science, Assuit University, Assuit 71516, Egypt \\ e-mail: rr_rashwan54@yahoo.com \\ * Corresponding author
}

\begin{abstract}
The aim of this paper is to prove a coincidence point theorem for a class of self mappings satisfying nonexpansive type condition under various conditions and a fixed point theorem is also obtained. Our results extend and generalize the corresponding result of Singh and Chandrashekhar [7].
\end{abstract}

\section{Introduction and Preliminaries}

The concept of 2-metric space was introduced by Gähler [2, 3, 4] whose abstract properties were suggested by the area function in Euclidean space. Employing various contractive conditions Iséki [5] setout the tradition of proving fixed point theorems in 2-metric spaces. Later on, Naidu and Prasad [6] contributed few fixed point theorems in 2-metric spaces introducing the concept of weak commutativity. Recently, Singh and Chandrashekhar [7] proved a fixed point theorem in 2-metric space for nonexpansive

Received: May 4, 2019; Accepted: June 6, 2019

2010 Mathematics Subject Classification: 47H10, 54H25.

Keywords and phrases: coincidence point, fixed point, nonexpansive type mapping, 2-metric space.

Copyright @ 2019 H. A. Hammad, A. H. Ansari and R. A. Rashwan. This is an open access article distributed under the Creative Commons Attribution License, which permits unrestricted use, distribution, and reproduction in any medium, provided the original work is properly cited. 
type mappings. They obtained the following result:

Theorem 1.1. Let $(X, d)$ be a 2-metric space and $T: X \rightarrow X$ be a self mapping satisfying the following nonexpansive type condition:

$$
\begin{aligned}
& d(T x, T y, u) \\
\leq & a \max \left\{d(x, y, u), d(x, T x, u), d(y, T y, u), \frac{1}{2}[d(x, T y, u)+d(y, T x, u)]\right\} \\
& +b \max \{d(x, T x, u), d(y, T y, u)\}+c[d(x, T y, u)+d(y, T x, u)],
\end{aligned}
$$

for all $x, y, u \in X$, where $a, b, c$ are real numbers such that $a+b+2 c=1$ and $a \geq 0, b>0, c>0$. Then $T$ has a unique fixed point and $T$ is continuous at the fixed point.

Our condition is an extension of that of Ćirić [1] (see also [8]). Also, we will show that our condition (2) includes the above condition (1).

Now we give some definitions which are used frequently to prove our main results.

Definition 1.1. Gähler defined 2-metric space as follows:

A 2-metric on a set $X$ with at least three points is a non-negative real-valued mapping $d: X \times X \times X \rightarrow R$ satisfying the following properties:

(1) To each pair of points $a, b$ with $a \neq b$ in $X$ there is a point $c \in X$ such that $d(a, b, c) \neq 0$.

(2) $d(a, b, c)=0$, if at least two of the points are equal,

(3) $d(a, b, c)=d(b, c, a)=d(a, c, b)$,

(4) $d(a, b, c) \leq d(a, b, u)+d(a, u, c)+d(u, b, c)$ for all $a, b, c, u \in X$.

The pair $(X, d)$ is called a 2-metric space.

Definition 1.2. The sequence $\left\{x_{n}\right\}$ is convergent to $x \in X$ and $x$ is the limit of this sequence if $\lim _{n \rightarrow \infty} d\left(x_{n}, x, u\right)=0$ for each $u \in X$.

Definition 1.3. A sequence $\left\{x_{n}\right\}$ is called Cauchy sequence if $\lim _{n, m \rightarrow \infty} d\left(x_{n}, x_{m}, u\right)=0$ for all $u \in X$. A 2-metric space in which every Cauchy sequence is convergent is called complete. 
Definition 1.4. Let $f$ and $g$ be two self mappings of a 2-metric space $(X, d)$. Then $f$ and $g$ are said to be compatible if $\lim _{n \rightarrow \infty} d\left(f g x_{n}, g f x_{n}, u\right)=0$ for each $u \in X$, whenever $\left\{x_{n}\right\}$ is a sequence such that $\lim _{n \rightarrow \infty} f x_{n}=\lim _{n \rightarrow \infty} g x_{n}=t \in X$.

Let $\Psi$ be a set of all continuous functions $\psi: \mathbb{R}_{+} \rightarrow \mathbb{R}_{+}$satisfying the following conditions:

$\left(\psi_{1}\right) \psi$ is continuous and strictly increasing.

$\left(\psi_{2}\right) \psi(t)=0$ if and only of $t=0$.

Let $\Phi_{u}$ be a set of all continuous functions $\varphi: \mathbb{R}_{+} \rightarrow \mathbb{R}_{+}$satisfying the following conditions:

$\left(\varphi_{1}\right) \varphi$ is continuous.

$\left(\varphi_{2}\right) \varphi(t)>0$ if $t>0$ and $\varphi(0) \geq 0$.

Let $\Phi$ be set of all lower continuous functions $\phi: \mathbb{R}_{+} \rightarrow \mathbb{R}_{+}$, such $\phi(t)=0$ if and only if $t=0$ and $\phi(t)<t$ for all $t>0$.

In 2014, Ansari [10] introduced the concept of $C$-class functions which cover a large class of contractive conditions.

Definition 1.5 [10]. Let $F: \mathbb{R}_{+}^{2} \rightarrow \mathbb{R}$ be a continuous mapping. Then it is called a C-class function if it satisfies the following conditions:

$\left(F_{1}\right) F(s, t) \leq s$, for all $(s, t) \in \mathbb{R}_{+}^{2}$.

$\left(F_{2}\right) \quad F(s, t)=s$ implies that $s=0$, or $t=0$, for all $(s, t) \in \mathbb{R}_{+}^{2}$.

Note for some $F$ we have that $F(0,0)=0$.

We denote $C$-class functions as $\mathcal{C}$.

Example 1.1 [10]. The following functions $F:[0, \infty)^{2} \rightarrow \mathbb{R}$ are elements of $\mathcal{C}$, for all $s, t \in[0, \infty)$ :

(1) $F(s, t)=s-t, F(s, t)=s \Rightarrow t=0$; 
(2) $F(s, t)=m s, 0<m<1, F(s, t)=s \Rightarrow s=0$;

(3) $F(s, t)=\frac{s}{(1+t)^{r}} ; r \in(0, \infty), F(s, t)=s \Rightarrow s=0$ or $t=0$;

(4) $F(s, t)=\log \left(t+a^{s}\right) /(1+t), a>1, F(s, t)=s \Rightarrow s=0$ or $t=0$;

(5) $F(s, t)=\ln \left(1+a^{s}\right) / 2, a>e, F(s, 1)=s \Rightarrow s=0$;

(6) $F(s, t)=(s+l)^{\left(1 /(1+t)^{r}\right)}-l, l>1, r \in(0, \infty), F(s, t)=s \Rightarrow t=0$;

(7) $F(s, t)=s \log _{t+a} a, a>1, F(s, t)=s \Rightarrow s=0$ or $t=0$;

(8) $F(s, t)=s-\left(\frac{1+s}{2+s}\right)\left(\frac{t}{1+t}\right), F(s, t)=s \Rightarrow t=0$;

(9) $F(s, t)=s \beta(s), \beta:[0, \infty) \rightarrow[0,1)$, and is continuous, $F(s, t)=s \Rightarrow s=0$;

(10) $F(s, t)=s-\frac{t}{k+t}, F(s, t)=s \Rightarrow t=0$;

(11) $\quad F(s, t)=s-\varphi(s), \quad F(s, t)=s \Rightarrow s=0$, here $\varphi:[0, \infty) \rightarrow[0, \infty)$ is a continuous function such that $\varphi(t)=0 \Leftrightarrow t=0$;

(12) $F(s, t)=\operatorname{sh}(s, t), F(s, t)=s \Rightarrow s=0$; here $h:[0, \infty) \times[0, \infty) \rightarrow[0, \infty)$ is a continuous function such that $h(t, s)<1$ for all $t, s>0$;

(13) $F(s, t)=s-\left(\frac{2+t}{1+t}\right) t, F(s, t)=s \Rightarrow t=0$.

(14) $F(s, t)=\sqrt[n]{\ln \left(1+s^{n}\right)}, F(s, t)=s \Rightarrow s=0$;

(15) $\quad F(s, t)=\phi(s), \quad F(s, t)=s \Rightarrow s=0$, here $\phi:[0, \infty) \rightarrow[0, \infty)$ is a upper semicontinuous function such that $\phi(0)=0$, and $\phi(t)<t$ for $t>0$,

(16) $F(s, t)=\frac{s}{(1+s)^{r}} ; r \in(0, \infty), F(s, t)=s \Rightarrow s=0$; 
(17) $F(s, t)=\vartheta(s) ; \vartheta: \mathbb{R}^{+} \times \mathbb{R}^{+} \rightarrow \mathbb{R}$ is a generalized Mizoguchi-Takahashi type function, $F(s, t)=s \Rightarrow s=0$;

(18) $F(s, t)=\frac{s}{\Gamma(1 / 2)} \int_{0}^{\infty} \frac{e^{-x}}{\sqrt{x}+t} d x$, where $\Gamma$ is the Euler Gamma function.

Let $\Psi$ be a set of all non-decreasing continuous functions $\psi: \mathbb{R}_{+} \rightarrow \mathbb{R}_{+}$, such $\psi(0)=0$.

Let $\Phi$ be a set of all continuous functions $\phi: \mathbb{R}_{+} \rightarrow \mathbb{R}_{+}$, such $\phi(0) \geq 0$.

Let $\Phi_{u}$ denote the class of the functions $\varphi:[0, \infty) \rightarrow[0, \infty)$ which satisfy the following conditions:

(a) $\varphi$ continuous;

(b) $\varphi(t)>0, t>0$ and $\varphi(0) \geq 0$.

Let $\Psi$ be a set of all continuous functions $\psi: \mathbb{R}_{+} \rightarrow \mathbb{R}_{+}$satisfying the following conditions:

$\left(\psi_{1}\right) \psi$ is continuous and strictly increasing.

$\left(\psi_{2}\right) \psi(t)=0$ if and only of $t=0$.

In this paper, we introduce a new class of self mappings satisfying the following nonexpansive type condition:

$$
\begin{aligned}
& \psi(d(T x, T y, u))
\end{aligned}
$$

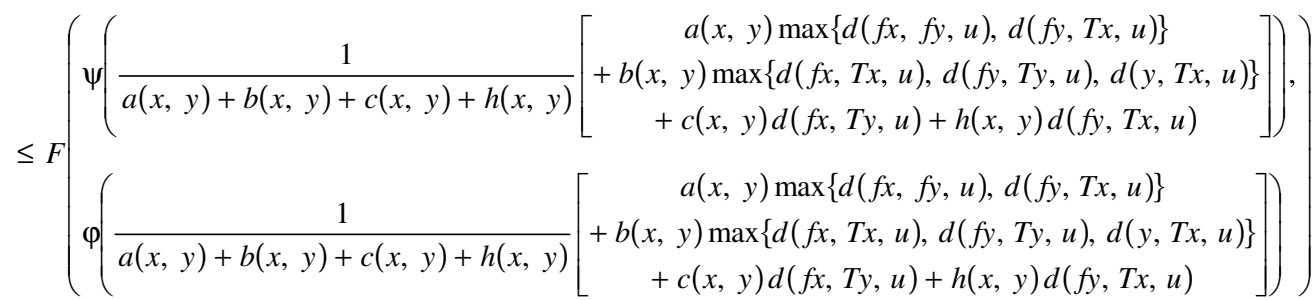

for all $x, y, u \in X$, where $\varphi \in \Phi_{u}, \psi \in \Psi, F \in \mathbb{C}$ and $a(x, y)+b(x, y)+c(x, y)$ $+h(x, y)>0$ and $h(x, y) \geq c(x, y)$. 


\section{Main Results}

Theorem 2.1. Let $(X, d)$ be a 2-metric space. Let $T, f$ be self mappings of $X$ satisfying nonexpansive type condition (2). Let $T(X) \subseteq f(X)$ and either

(a) $X$ is complete and $f$ is surjective, or,

(b) $X$ is complete, $f$ is continuous and the pair $(T, f)$ is compatible.

(c) Either $f(X)$ is complete, or $T(X)$.

Then $f$ and $T$ have a coincidence point in X. Further, the coincidence point is unique, that is, $f_{p}=f_{q}$, whenever $f_{p}=T_{p}$ and $f_{q}=T_{q} ; p, q \in X$.

Proof. Let $x=x_{0}$ be an arbitrary point in $X$. Since $T(X) \subseteq f(X)$, choose $x_{1}$ so that $y_{1}=f x_{1}=T x_{0}$. In general, choose $x_{n+1}$ such that $y_{n+1}=f x_{n+1}=T x_{n}$ for all $n=0,1,2, \ldots$.

On applying inequality (2) and taking $a\left(x_{n}, x_{n+1}\right)=a, \quad b\left(x_{n}, x_{n+1}\right)=b$, $c\left(x_{n}, x_{n+1}\right)=c$ and $h\left(x_{n}, x_{n+1}\right)=h$, we get

$$
\begin{aligned}
& \psi\left(d\left(f x_{n+2}, f x_{n+1}, f x_{n}\right)\right) \\
= & \psi\left(d\left(T x_{n+1}, T x_{n}, f x_{n}\right)\right)
\end{aligned}
$$$$
\leq F\left(\psi\left(\begin{array}{c}
\left.\frac{1}{a+b+c+h}\left[\begin{array}{c}
a \max \left\{d\left(f x_{n+1}, f x_{n}, f x_{n}\right), d\left(f x_{n+1}, T x_{n+1}, f x_{n}\right)\right\} \\
+b \max \left\{d\left(f x_{n+1}, T x_{n+1}, f x_{n}\right), d\left(f x_{n}, T x_{n}, f x_{n}\right), d\left(f x_{n}, T x_{n+1}, f x_{n}\right)\right\} \\
+c d\left(f x_{n+1}, T x_{n}, f x_{n}\right)+h d\left(f x_{n}, T x_{n+1}, f x_{n}\right)
\end{array}\right]\right), \\
a \max \left\{d\left(f x_{n+1}, f x_{n}, f x_{n}\right), d\left(f x_{n+1}, T x_{n+1}, f x_{n}\right)\right\} \\
\left.\frac{1}{a+b+c+h}\left[\begin{array}{c}
+b \max \left\{d\left(f x_{n+1}, T x_{n+1}, f x_{n}\right), d\left(f x_{n}, T x_{n}, f x_{n}\right), d\left(f x_{n}, T x_{n+1}, f x_{n}\right)\right\} \\
+c d\left(f x_{n+1}, T x_{n}, f x_{n}\right)+h d\left(f x_{n}, T x_{n+1}, f x_{n}\right)
\end{array}\right]\right)
\end{array}\right),\right.
$$$$
=F\left(\psi\left(\frac{a+b}{a+b+c+h} d\left(f x_{n+1}, T x_{n+1}, f x_{n}\right)\right), \varphi\left(\frac{a+b}{a+b+c+h} d\left(f x_{n+1}, T x_{n+1}, f x_{n}\right)\right)\right)
$$$$
=F\left(\psi\left(\frac{a+b}{a+b+c+h} d\left(f x_{n+2}, f x_{n+1}, f x_{n}\right)\right), \varphi\left(\frac{a+b}{a+b+c+h} d\left(f x_{n+2}, f x_{n+1}, f x_{n}\right)\right)\right)
$$$$
\leq \psi\left(\frac{a+b}{a+b+c+h} d\left(f x_{n+2}, f x_{n+1}, f x_{n}\right)\right) \leq \psi\left(d\left(f x_{n+2}, f x_{n+1}, f x_{n}\right)\right) .
$$ 
So,

$$
\psi\left(\frac{a+b}{a+b+c+h} d\left(f x_{n+2}, f x_{n+1}, f x_{n}\right)\right)=0
$$

or

$$
\varphi\left(\frac{a+b}{a+b+c+h} d\left(f x_{n+2}, f x_{n+1}, f x_{n}\right)\right)=0
$$

thus

$$
d\left(f x_{n+2}, f x_{n+1}, f x_{n}\right)=0 .
$$

On applying inequality (2) again and using triangular inequality and (3), we get

$$
\psi\left(d\left(T x_{n}, T x_{n+1}, u\right)\right)
$$$$
\leq F\left(\psi \left(\begin{array}{c}
a \max \left\{d\left(f x_{n}, f x_{n+1}, u\right), d\left(f x_{n+1}, T x_{n}, u\right)\right\} \\
\left.\frac{1}{a+b+c+h}\left[\begin{array}{c}
a \max \left\{d\left(f x_{n}, T x_{n}, u\right), d\left(f x_{n+1}, T x_{n+1}, u\right), d\left(f x_{n+1}, T x_{n}, u\right)\right\} \\
+c d\left(f x_{n}, T x_{n+1}, u\right)+h d\left(f x_{n+1}, T x_{n}, u\right)
\end{array}\right]\right), \\
\varphi\left(\begin{array}{c}
a \max \left\{d\left(f x_{n}, f x_{n+1}, u\right), d\left(f x_{n+1}, T x_{n}, u\right)\right\} \\
\left.\frac{1}{a+b+c+h}\left[\begin{array}{c}
+b \max \left\{d\left(f x_{n}, T x_{n}, u\right), d\left(f x_{n+1}, T x_{n+1}, u\right), d\left(f x_{n+1}, T x_{n}, u\right)\right\} \\
+c d\left(f x_{n}, T x_{n+1}, u\right)+h d\left(f x_{n+1}, T x_{n}, u\right)
\end{array}\right]\right)
\end{array}\right),
\end{array}\right.\right.
$$$$
=F\left(\begin{array}{c}
\left.\frac{1}{a+b+c+h}\left[\begin{array}{c}
a \max \left\{d\left(f x_{n}, T x_{n}, u\right), d\left(f x_{n+1}, T x_{n+1}, u\right)\right\} \\
+b \max \left\{d\left(f x_{n}, T x_{n}, u\right), d\left(f x_{n+1}, T x_{n+1}, u\right)\right\} \\
+c d\left(f x_{n}, T x_{n+1}, T x_{n}\right)+c d\left(f x_{n}, T x_{n}, u\right) \\
+c d\left(T x_{n+1}, T x_{n}, u\right)
\end{array}\right]\right) \\
\varphi\left(\frac{1}{a+b+c+h}\left[\begin{array}{c}
a \max \left\{d\left(f x_{n}, T x_{n}, u\right), d\left(f x_{n+1}, T x_{n+1}, u\right)\right\} \\
+b \max \left\{d\left(f x_{n}, T x_{n}, u\right), d\left(f x_{n+1}, T x_{n+1}, u\right)\right\} \\
+c d\left(f x_{n}, T x_{n+1}, T x_{n}\right)+c d\left(f x_{n}, T x_{n}, u\right) \\
+c d\left(T x_{n+1}, T x_{n}, u\right)
\end{array}\right]\right) \\
\left.\left[\begin{array}{c}
1 \\
+
\end{array}\right]\right)
\end{array}\right)
$$

$$
=F\left(\begin{array}{c}
\psi\left(\frac{1}{a+b+c+h}\left[\begin{array}{c}
a \max \left\{d\left(f x_{n}, T x_{n}, u\right), d\left(f x_{n+1}, T x_{n+1}, u\right)\right\} \\
+b \max \left\{d\left(f x_{n}, T x_{n}, u\right), d\left(f x_{n+1}, T x_{n+1}, u\right)\right\} \\
+c\left[d\left(f x_{n}, T x_{n}, u\right)+d\left(f x_{n+1}, T x_{n+1}, u\right)\right]
\end{array}\right]\right) \\
\varphi\left(\frac{1}{a+b+c+h}\left[\begin{array}{c}
a \max \left\{d\left(f x_{n}, T x_{n}, u\right), d\left(f x_{n+1}, T x_{n+1}, u\right)\right\} \\
+b \max \left\{d\left(f x_{n}, T x_{n}, u\right), d\left(f x_{n+1}, T x_{n+1}, u\right)\right\} \\
+c\left[d\left(f x_{n}, T x_{n}, u\right)+d\left(f x_{n+1}, T x_{n+1}, u\right)\right]
\end{array}\right]\right)
\end{array}\right)
$$


$\leq \psi\left(\frac{1}{a+b+c+h}\left[\begin{array}{c}a \max \left\{d\left(f x_{n}, T x_{n}, u\right), d\left(f x_{n+1}, T x_{n+1}, u\right)\right\} \\ +b \max \left\{d\left(f x_{n}, T x_{n}, u\right), d\left(f x_{n+1}, T x_{n+1}, u\right)\right\} \\ +c\left[d\left(f x_{n}, T x_{n}, u\right)+d\left(f x_{n+1}, T x_{n+1}, u\right)\right]\end{array}\right]\right)$.

Suppose that, for some $n, d\left(f x_{n+1}, T x_{n+1}, u\right)>d\left(f x_{n}, T x_{n}, u\right)$, then from (3), we have

$$
\begin{aligned}
& \psi\left(d\left(f x_{n+1}, T x_{n+1}, u\right)\right) \\
= & \psi\left(d\left(T x_{n}, T x_{n+1}, u\right)\right) \\
\leq & F\left(\begin{array}{c}
\psi\left(\frac{1}{a+b+c+h}\left[\begin{array}{c}
a d\left(f x_{n+1}, T x_{n+1}, u\right)+b d\left(f x_{n+1}, T x_{n+1}, u\right) \\
+c\left[d\left(f x_{n+1}, T x_{n+1}, u\right)+d\left(f x_{n+1}, T x_{n+1}, u\right)\right]
\end{array}\right]\right), \\
\varphi\left(\frac{1}{a+b+c+h}\left[\begin{array}{c}
a d\left(f x_{n+1}, T x_{n+1}, u\right)+b d\left(f x_{n+1}, T x_{n+1}, u\right) \\
+c\left[d\left(f x_{n+1}, T x_{n+1}, u\right)+d\left(f x_{n+1}, T x_{n+1}, u\right)\right]
\end{array}\right]\right)
\end{array}\right) \\
\leq & \psi\left(\frac{1}{a+b+c+h}\left[\begin{array}{c}
a d\left(f x_{n+1}, T x_{n+1}, u\right)+b d\left(f x_{n+1}, T x_{n+1}, u\right) \\
+c\left[d\left(f x_{n+1}, T x_{n+1}, u\right)+d\left(f x_{n+1}, T x_{n+1}, u\right)\right]
\end{array}\right]\right) \\
= & \psi\left(\frac{a+b+2 c}{a+b+c+h} d\left(f x_{n+1}, T x_{n+1}, u\right)\right) \\
\leq & \psi\left(d\left(f x_{n+1}, T x_{n+1}, u\right)\right) .
\end{aligned}
$$

So,

$$
\psi\left(\frac{a+b}{a+b+c+h} d\left(f x_{n+2}, f x_{n+1}, u\right)\right)=0
$$

or

$$
\varphi\left(\frac{a+b}{a+b+c+h} d\left(f x_{n+2}, f x_{n+1}, u\right)\right)=0,
$$

thus $d\left(f x_{n+2}, f x_{n+1}, u\right)=0$, a contradiction. Hence we must have, $d\left(f x_{n+1}, T x_{n+1}, u\right)$ $\leq d\left(f x_{n}, T x_{n}, u\right)$, or equivalently,

$$
d\left(T x_{n}, T x_{n+1}, u\right) \leq d\left(T x_{n-1}, T x_{n}, u\right) .
$$

On applying inequality (2) again and evaluating $a, b, c$ at $\left(x_{n-1}, x_{n}\right)$, we have

$$
\begin{aligned}
& \psi\left(d\left(y_{n}, y_{n+1}, u\right)\right) \\
= & \psi\left(d\left(T x_{n-1}, T x_{n}, u\right)\right)
\end{aligned}
$$




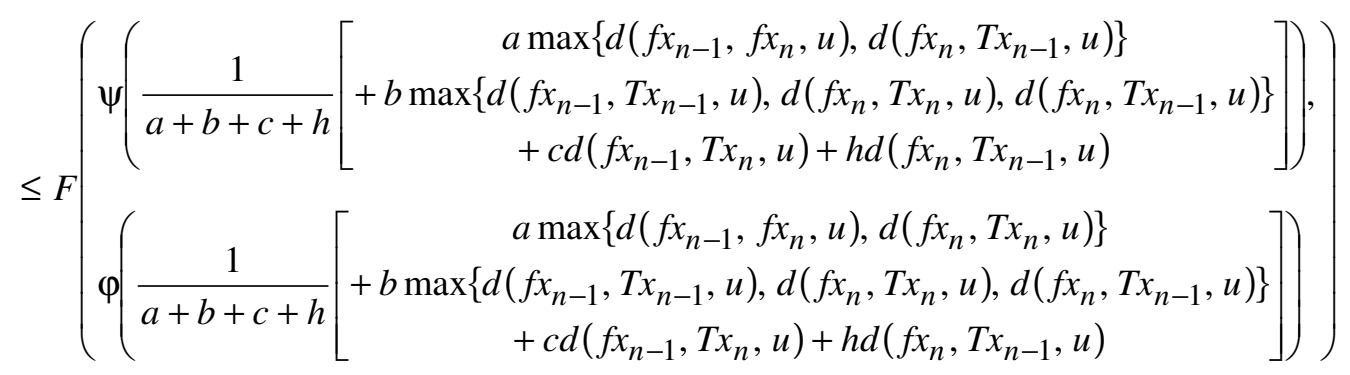

$=F\left(\begin{array}{c}\psi\left(\frac{1}{a+b+c+h}\left[\begin{array}{c}a \max \left\{d\left(T x_{n-2}, T x_{n-1}, u\right), d\left(T x_{n-1}, T x_{n}, u\right)\right\} \\ +b \max \left\{d\left(T x_{n-2}, T x_{n-1}, u\right), d\left(T x_{n-1}, T x_{n}, u\right)\right\} \\ +c d\left(T x_{n-2}, T x_{n}, u\right)\end{array}\right]\right), \\ \left.\varphi\left(\begin{array}{c}a \max \left\{d\left(T x_{n-2}, T x_{n-1}, u\right), d\left(T x_{n-1}, T x_{n}, u\right)\right\} \\ +b \max \left\{d\left(T x_{n-2}, T x_{n-1}, u\right), d\left(T x_{n-1}, T x_{n}, u\right)\right\} \\ +c d\left(T x_{n-2}, T x_{n}, u\right)\end{array}\right]\right)\end{array}\right)$

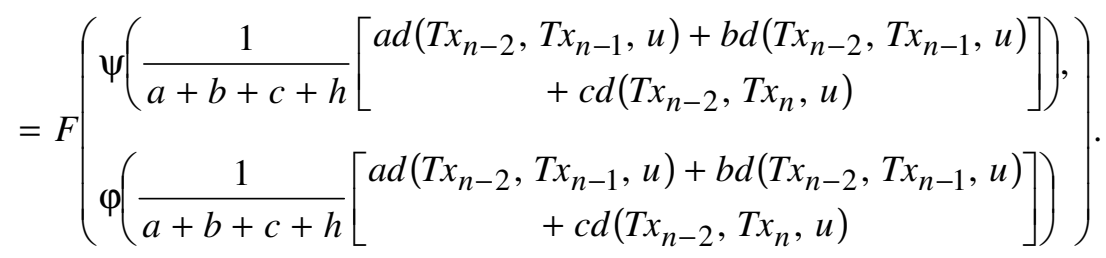

On applying inequality (2) again and using (3), (5) and by triangular inequality, we get $\psi\left(d\left(T x_{n-2}, T x_{n}, u\right)\right)$

$\leq F\left(\psi\left(\begin{array}{c}\left.\frac{1}{\bar{a}+\bar{b}+\bar{c}+\bar{h}}\left[\begin{array}{c}\bar{a} \max \left\{d\left(f x_{n-2}, f x_{n}, u\right), d\left(f x_{n}, T x_{n}, u\right)\right\} \\ +\bar{b} \max \left\{d\left(f x_{n-2}, T x_{n-2}, u\right), d\left(f x_{n}, T x_{n}, u\right), d\left(f x_{n}, T x_{n-2}, u\right)\right\} \\ +\bar{c}\left[d\left(f x_{n-2}, T x_{n}, u\right)+\bar{h} d\left(f x_{n}, T x_{n-2}, u\right)\right]\end{array}\right]\right), \\ \varphi(\cdots)\end{array}\right)\right.$

$=F\left(\psi\left(\begin{array}{c}\frac{1}{\bar{a} \max \left\{d\left(T x_{n-3}, T x_{n-1}, u\right), d\left(T x_{n-1}, T x_{n}, u\right)\right\}} \\ \left.\bar{a}+\bar{b}+\bar{c}+\bar{h}\left[\begin{array}{c} \\ +\bar{b} \max \left\{d\left(T x_{n-3}, T x_{n-2}, u\right), d\left(T x_{n-1}, T x_{n}, u\right), d\left(T x_{n-1}, T x_{n-2}, u\right)\right\} \\ +\bar{c}\left[d\left(T x_{n-3}, T x_{n}, u\right)+d\left(T x_{n-1}, T x_{n-2}, u\right)\right]\end{array}\right]\right), \\ \varphi(\cdots)\end{array}\right)\right.$ 


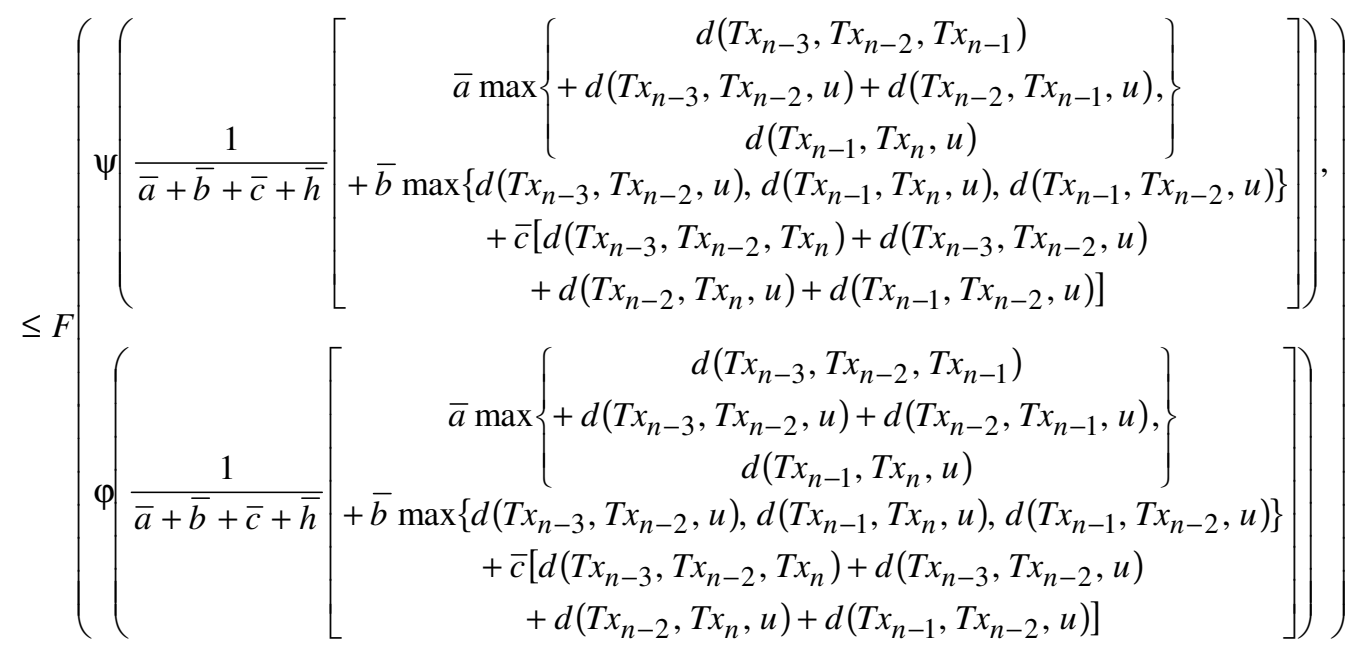

$\leq F\left(\psi\left(\left[\begin{array}{c}\bar{a} \max \left\{\begin{array}{c}d\left(T x_{n-3}, T x_{n-2}, T x_{n-1}\right)+d\left(T x_{n-3}, T x_{n-2}, u\right) \\ +d\left(T x_{n-2}, T x_{n-1}, u\right), d\left(T x_{n-1}, T x_{n}, u\right)\end{array}\right\} \\ \left.\bar{a}+\bar{b}+\bar{c}+\bar{h}\left[\begin{array}{c}\bar{b} \max \left\{d\left(T x_{n-3}, T x_{n-2}, u\right), d\left(T x_{n-1}, T x_{n}, u\right), d\left(T x_{n-1}, T x_{n-2}, u\right)\right\} \\ +\bar{c}\left[d\left(T x_{n-3}, T x_{n-2}, T x_{n-1}\right)+d\left(T x_{n-3}, T x_{n-1}, T x_{n}\right)\right. \\ \left.+d\left(T x_{n-2}, T x_{n-1}, T x_{n}\right)+d\left(T x_{n-3}, T x_{n-2}, u\right)\right]\end{array}\right]\right), \\ \varphi(\cdots)\end{array}\right)\right)\right.$

$=F\left(\psi\left(\frac{1}{\bar{a}+\bar{b}+\bar{c}+\bar{h}}\left[\begin{array}{c}\bar{a} \max \left\{d\left(T x_{n-3}, T x_{n-2}, u\right)+d\left(T x_{n-2}, T x_{n-1}, u\right), d\left(T x_{n-1}, T x_{n}, u\right)\right\} \\ +\bar{b} \max \left\{d\left(T x_{n-3}, T x_{n-2}, u\right), d\left(T x_{n-1}, T x_{n}, u\right), d\left(T x_{n-1}, T x_{n-2}, u\right)\right\} \\ +\bar{c}\left[d\left(T x_{n-3}, T x_{n-1}, T x_{n}\right)+d\left(T x_{n-3}, T x_{n-2}, u\right)\right. \\ \left.+d\left(T x_{n}, T x_{n-1}, u\right)+d\left(T x_{n-2}, T x_{n-1}, u\right)\right] \\ \varphi(\cdots)\end{array}\right)\right.\right.$,

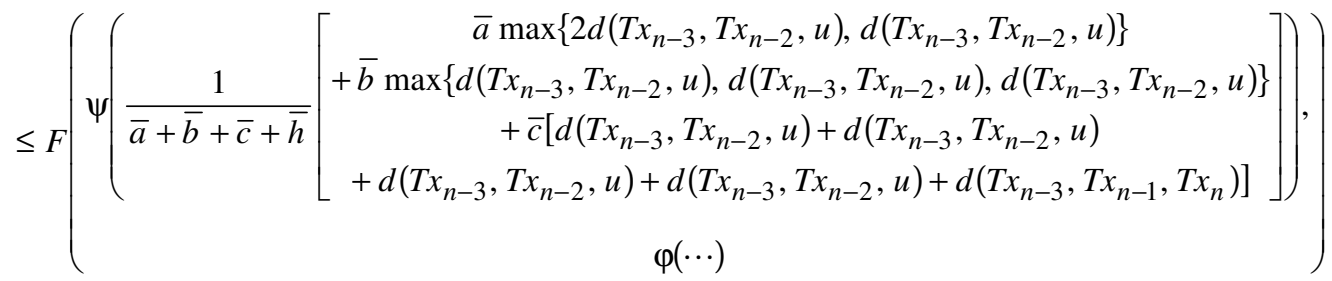

$\leq F\left(\psi\left(\frac{2(\bar{a}+\bar{b}+\bar{c})-\bar{b}}{\bar{a}+\bar{b}+\bar{c}+\bar{h}} d\left(T x_{n-3}, T x_{n-2}, u\right)\right), \varphi\left(\frac{1}{\bar{a}+\bar{b}+\bar{c}+\bar{h}} d\left(T x_{n-3}, T x_{n-2}, u\right)\right)\right)$

$\leq F\left(\psi\left(\frac{2-\bar{b}}{\bar{a}+\bar{b}+\bar{c}+\bar{h}}\left(T x_{n-3}, T x_{n-2}, u\right)\right), \varphi\left(\frac{1}{\bar{a}+\bar{b}+\bar{c}+\bar{h}} d\left(T x_{n-3}, T x_{n-2}, u\right)\right)\right)$. 
This implies that

$$
\begin{aligned}
\psi\left(d\left(T x_{n-2}, T x_{n}, u\right)\right) \leq F\left(\psi\left(\frac{2-\bar{b}}{\bar{a}+\bar{b}+\bar{c}+\bar{h}} d\left(T x_{n-3}, T x_{n-2}, u\right)\right)\right. \\
\left.\varphi\left(\frac{1}{\bar{a}+\bar{b}+\bar{c}+\bar{h}} d\left(T x_{n-3}, T x_{n-2}, u\right)\right)\right) \\
\leq \psi\left(\frac{2-\bar{b}}{\bar{a}+\bar{b}+\bar{c}+\bar{h}} d\left(T x_{n-3}, T x_{n-2}, u\right)\right),
\end{aligned}
$$

where $\bar{a}, \bar{b}, \bar{c}$ are evaluated at $\left(x_{n-2}, x_{n}\right)$.

At the bottom line of the above inequality, $d\left(T x_{n-3}, T x_{n-1}, T x_{n}\right)=0$.

Because, let $d\left(T x_{n-3}, T x_{n-1}, T x_{n}\right) \neq 0$, then applying (4), we get

$$
\begin{aligned}
d\left(T x_{n-3}, T x_{n-1}, T x_{n}\right)= & d\left(T x_{n-1}, T x_{n}, T x_{n-3}\right) \\
\leq & a \max \left\{d\left(f x_{n-1}, T x_{n-1}, T x_{n-3}\right), d\left(f x_{n}, T x_{n}, T x_{n-3}\right)\right\} \\
& +b \max \left\{d\left(f x_{n-1}, T x_{n-1}, T x_{n-3}\right), d\left(f x_{n}, T x_{n}, T x_{n-3}\right)\right\} \\
& +c\left[d\left(f x_{n-1}, T x_{n-1}, T x_{n-3}\right)+d\left(f x_{n}, T x_{n}, T x_{n-3}\right)\right] \\
\leq & a \max \left\{d\left(T x_{n-2}, T x_{n-1}, T x_{n-3}\right), d\left(T x_{n-1}, T x_{n}, T x_{n-3}\right)\right\} \\
& +b \max \left\{d\left(T x_{n-2}, T x_{n-1}, T x_{n-3}\right), d\left(T x_{n-1}, T x_{n}, T x_{n-3}\right)\right\} \\
& +c\left[d\left(T x_{n-2}, T x_{n-1}, T x_{n-3}\right)+d\left(T x_{n-1}, T x_{n}, T x_{n-3}\right)\right] \\
= & (a+b+c) d\left(T x_{n-1}, T x_{n}, T x_{n-3}\right) \\
< & d\left(T x_{n-1}, T x_{n}, T x_{n-3}\right) .
\end{aligned}
$$

A contradiction. Thus, $d\left(T x_{n-3}, T x_{n-1}, T x_{n}\right)=0$.

Using (5), (5) and (6), we get

$$
\begin{aligned}
& d\left(T x_{n-1}, T x_{n}, u\right) \\
= & d\left(y_{n}, y_{n+1}, u\right) \\
\leq & a d\left(T x_{n-2}, T x_{n-1}, u\right)+b d\left(T x_{n-2}, T x_{n-1}, u\right)+c\left[(2-\bar{b}) d\left(T x_{n-3}, T x_{n-2}, u\right)\right]
\end{aligned}
$$




$$
\begin{aligned}
& \leq a d\left(T x_{n-3}, T x_{n-2}, u\right)+b d\left(T x_{n-3}, T x_{n-2}, u\right)+c(2-\bar{b}) d\left(T x_{n-3}, T x_{n-2}, u\right) \\
& =(a+b+2 c) d\left(T x_{n-3}, T x_{n-2}, u\right)-\bar{b} c d\left(T x_{n-3}, T x_{n-2}, u\right) \\
& \leq(1-\bar{b} c) d\left(T x_{n-3}, T x_{n-2}, u\right) \\
& \leq(1-\beta \gamma) d\left(T x_{n-3}, T x_{n-2}, u\right) \\
& \leq(1-\beta \gamma) \frac{n}{2} d\left(y_{0}, y_{1}, u\right) .
\end{aligned}
$$

Hence $\left\{y_{n}\right\}$ is a Cauchy sequence.

For case (a) and (b), suppose that $X$ is complete. Then Cauchy sequence $\left\{y_{n}\right\}$ will converge to a point $p$ in $X$.

Case (a): Since $f$ is surjective, there exists a point $z$ in $X$ such that $p=f z$.

Now applying inequality (2), we get

$$
d(f z, T z, u)
$$$$
\leq d\left(f z, y_{n+1}, u\right)+d\left(f z, T z, y_{n+1}\right)+d\left(T z, y_{n+1}, u\right)
$$$$
\leq d\left(f z, y_{n+1}, u\right)+d\left(f z, T z, y_{n+1}\right)+d\left(T x_{n}, T z, u\right)
$$$$
\leq d\left(f z, y_{n+1}, u\right)+d\left(f z, T z, y_{n+1}\right)+a(x, y) \max \left\{d\left(f x_{n}, f z, u\right), d(f z, T z, u)\right\}
$$$$
+b(x, y) \max \left\{d\left(f x_{n}, T x_{n}, u\right), d(f z, T z, u), d\left(f z, T x_{n}, u\right)\right\}
$$$$
+c(x, y)\left[d\left(f x_{n}, T z, u\right)+d\left(f z, T x_{n}, u\right)\right]
$$

$\leq \sup \{a(x, y)+c(x, y)\} \cdot \max \left\{\max \left\{d\left(f x_{n}, f z, u\right), d(f z, T z, u)\right\}, d\left(f z, f x_{n+1}, u\right)\right\}$ $x, y \in X$

$$
+\sup _{x, y \in X}\{b(x, y)+c(x, y)\} \cdot \max \left\{\begin{array}{c}
\max \left\{d\left(f x_{n}, f x_{n+1}, u\right), d(f z, T z, u), d\left(f z, f x_{n+1}, u\right)\right\}, \\
d\left(f x_{n}, T z, u\right)+d\left(f z, f x_{n+1}, u\right)
\end{array}\right\} .
$$

Taking the limit as $n \rightarrow \infty$, we have

$$
d(f z, T z, u) \leq \sup x, y \in X(b+c) d(f z, T z, u)<d(f z, T z, u),
$$

implies that $f z=T z$. 
Case (b): Since $f$ is continuous and $f, T$ are compatible, we have

$$
\lim _{n \rightarrow \infty} f y_{n}=f p, \lim _{n \rightarrow \infty} f x_{n}=\lim _{n \rightarrow \infty} T x_{n}=\lim _{n \rightarrow \infty} y_{n+1}=p,
$$

and hence

$$
\lim _{n \rightarrow \infty} d\left(f T x_{n}, T f x_{n}, u\right)=0 .
$$

By above results, we have

$$
\begin{aligned}
& d(f p, T p, u) \\
\leq & d\left(f p, f y_{n+1}, T p\right)+d\left(f p, f y_{n+1}, u\right)+d\left(f y_{n+1}, u, T p\right) \\
\leq & d\left(f p, f y_{n+1}, T p\right)+d\left(f p, f y_{n+1}, u\right)+d\left(T p, T f x_{n}, u\right) \\
\leq & d\left(f p, f y_{n+1}, T p\right)+d\left(f p, f y_{n+1}, u\right) \\
& +a \max \left\{d\left(f f x_{n}, f p, u\right), d(f p, T p, u)\right\} \\
& +b \max \left\{d\left(f f x_{n}, T f x_{n}, u\right), d(f p, T p, u), d\left(f p, T f x_{n}, u\right)\right\} \\
& +c\left[d\left(f f x_{n}, T p, u\right)+d\left(f p, T f x_{n}, u\right)\right] \\
\leq & d\left(f p, f y_{n+1}, T p\right)+d\left(f p, f y_{n+1}, u\right) \\
& +\sup _{x, y \in X}[a(x, y)+b(x, y)+c(x, y)]\left(\max \left\{\begin{array}{c}
d\left(f f x_{n}, T f x_{n}, u\right), d(f p, T p, u), \\
d\left(f p, T f x_{n}, u\right)
\end{array}\right\}\right. \\
& \left.+d\left(f f x_{n}, T f x_{n}, u\right), d(f p, T p, u), d\left(f p, T f x_{n}, u\right)\right) .
\end{aligned}
$$

Now we have

$$
d\left(f f x_{n}, T f x_{n}, u\right) \leq d\left(f f x_{n}, f T x_{n}, u\right)+d\left(f T x_{n}, T f x_{n}, u\right)+d\left(f f x_{n}, T f x_{n}, T f x_{n}\right) .
$$

Using the continuity of $f$ and the compatibility of $f$ and $T$, it follows that

$$
\lim _{n \rightarrow \infty} d\left(f f x_{n}, T f x_{n}, u\right)=0, \lim _{n \rightarrow \infty} d\left(f f x_{n}, f T x_{n}, u\right)=0 .
$$

$\lim _{n \rightarrow \infty} f f x_{n}=f p$, implies that $\lim _{n \rightarrow \infty} T f x_{n}=f p$. 
Taking limit as $n \rightarrow \infty$ and using the inequality (6) and (7), we get

$$
d(f p, T p, u) \leq \sup _{x, y \in X}[a(x, y)+b(x, y)+c(x, y)] d(f p, T p, u) \text {, implies that } f p=T p .
$$

Case (c): In this case, $p \in f(X)$. Let $z \in f^{-1} p$, then $p=f z$, and the proof is completed by Case (a).

To establish uniqueness, suppose that $q$ is another coincidence point of $f$ and $T$. Then from (2) with $a, b, c$ evaluated at $(p, q)$, we have

$$
\begin{aligned}
d(T p, T q, u) \leq & a \max \{d(f p, f q, u), d(f p, T q, u)\} \\
& +b \max \{d(f p, T p, u), d(f q, T q, u), d(f q, T p, u)\} \\
& +c[d(f p, T q, u)+d(f q, T p, u)] \\
\leq & (a+b+2 c) d(T p, T q, u) .
\end{aligned}
$$

Hence $T p=T q$.

Corollary 2.1. Let $(X, d)$ be a complete 2-metric space and $T$ be a self map of $X$ satisfying (2) with $f=I$, the identity mapping on $X$. Then $T$ has a unique fixed point and at this fixed point $T$ is continuous.

Proof. The existence and uniqueness of the fixed point comes from Theorem 2.1 by setting $f=I$. To prove continuity at the unique fixed point $p$, we apply inequality (2), where $a, b, c$ are evaluated at $\left(y_{n}, p\right)$.

$$
\begin{aligned}
d\left(T y_{n}, p, u\right)= & d\left(T y_{n}, T p, u\right) \\
\leq & a \max \left\{d\left(y_{n}, p, u\right), d(p, T p, u)\right\} \\
& +b \max \left\{d\left(y_{n}, T y_{n}, u\right), d(p, T p, u), d\left(p, T y_{n}, u\right)\right\} \\
& +c\left[d\left(y_{n}, T p, u\right)+d\left(p, T y_{n}, u\right)\right] .
\end{aligned}
$$

Taking limit as $n \rightarrow \infty$ yields

$$
\lim _{n \rightarrow \infty} d\left(T y_{n}, p, u\right) \leq(b+c) \lim _{n \rightarrow \infty} d\left(p, T y_{n}, u\right)<\lim _{n \rightarrow \infty} d\left(p, T y_{n}, u\right) .
$$

a contradiction. Therefore, $\lim _{n \rightarrow \infty} T y_{n}=p=T p$. 
Remark 2.1. Our condition (2) includes condition (1) of [7] if we define, with $f=I$ the identity mapping,

$$
m(x, y, u)=\max \left\{d(x, y, u), d(x, T x, u), d(y, T y, u), \frac{1}{2}[d(x, T y, u)+d(y, T x, u)]\right\} .
$$

For each $x, y \in X$ such that

$$
m(x, y, u)=\max \{d(x, T x, u), d(y, T y, u)\},
$$

define $a(x, y)=0, b(x, y)=a+b, c(x, y)=c$.

For each $x, y \in X$ such that

$$
m(x, y, u)=\frac{1}{2}[d(x, T y, u)+d(y, T x, u)]
$$

define $a(x, y)=0, b(x, y)=b, c(x, y)=a+2 c$.

Hence our Theorem 2.1 is a proper generalization of [7].

\section{References}

[1] Lj. B. Ćirić, On some nonexpansive type mappings and fixed points, Indian J. Pure Appl. Math. 24(3) (1993), 145-149.

[2] S. Gähler, Lineare 2-normierte Räume, Math. Nachr. 28 (1964), 1-43. https://doi.org/10.1002/mana.19640280102

[3] S. Gähler, 2-metrische Räume und ihre topologische Struktur, Math. Nachr. 26 (1963), 115-148. https://doi.org/10.1002/mana.19630260109

[4] S. Gähler, Über die Uniformisierbarkeit 2-metrischer Räume, Math. Nachr. 28 (1964), 235-244. https://doi.org/10.1002/mana.19640280309

[5] K. Iséki, Fixed point theorem in 2-metric spaces, Math. Sem. Notes Kobe Univ. 3(1) (1975), 133-136.

[6] S. V. R. Naidu and J. R. Prasad, Fixed point theorems in 2-metric spaces, Indian J. Pure Appl. Math. 17(8) (1986), 974-993.

[7] S. L. Singh and G. Chandrashekhar, A fixed point theorem in a 2-metric space and an application, J. Natur. Phys. Sci. 15(1-2) (2001), 55-64. 
[8] N. Chandra, S. N. Mishra, S. L. Singh and B. E. Rhoades, Coincidence and fixed points of nonexpansive type multi-valued and single-valued maps, Indian J. Pure Appl. Math. 26(5) (1995), 393-401.

[9] R. K. Namdeo, S. Dubey and K. Tas, Coincidence and fixed points of non-expansive type mappings on 2-metric spaces, Int. Math. Forum 2(17) (2007), 803-811. https://doi.org/10.12988/imf.2007.07071

[10] A. H. Ansari, Note on $\varphi-\psi$-contractive type mappings and related fixed point, The 2 nd Regional Conference on Mathematics and Applications, Payame Noor University, 2014, pp. 377-380. 